\title{
On the equivalence of the RTI and SVM approaches to time correlated analysis
}

\author{
S. Croft ${ }^{1 *}$, A. Favalli ${ }^{2}$, D. Henzlova ${ }^{2}$ and P.A. Santi ${ }^{2}$ \\ ${ }^{1}$ Safeguards \& Security Technology (SST), Global Nuclear Security Technology Division, PO Box 2008, Bldg \\ 5700, MS-6166, Oak Ridge, TN 37831-6166, USA \\ ${ }^{2}$ Safeguards Science \& Technology Group (NEN-1), Nuclear Engineering and Nonproliferation Division, MS-E540, \\ Los Alamos, NM 87545, USA
}

*Corresponding author. Email crofts@ornl.gov. Phone +1 (865)-241-2834

Recently two papers on how to perform passive neutron auto-correlation analysis on time gated histograms formed from pulse train data, generically called time correlation analysis (TCA), have appeared in this journal [1,2]. For those of us working in international nuclear safeguards these treatments are of particular interest because passive neutron multiplicity counting is a widely deployed technique for the quantification of plutonium. The purpose of this letter is to show that the skewness-variance-mean (SVM) approach developed in [1] is equivalent in terms of assay capability to the random trigger interval (RTI) analysis laid out in [2]. Mathematically we could also use other numerical ways to extract the time correlated information from the histogram data including, for example, what we might call the mean, mean square, and mean cube approach. The important feature however, from the perspective of real world applications, is that the correlated information extracted is the same, and subsequently gets interpreted in the same way based on the same underlying physics model.

Keywords

Time correlation analysis; Neutron multiplicity counting; Neutron auto-correlation analysis

We begin by recapping an important earlier result of Böhnel [3] who, under the assumptions of a point-like measurement item in which the neutron multiplication can be considered instantaneous when compared to the temporal response of the external detector (the so call superfission concept), derived closed-form expressions for the emergent reduced factorial moments by using probability generating function (PGF) theory. The corresponding observable rates are readily obtained. The formulation in terms of factorial moments is a natural choice in that the resulting equations for the self-perpetuating fission chains can be shown to converge whereas the number distribution extends to infinity. The expressions, referred to as the point model expressions, relate the observed multiplet rates to the generalized spontaneous fission source rate, the 
properties of the item (source strength and leakage self-multiplication), and detector (efficiency, gate utilization factors). In a practical setting under suitable conditions the first three multiplet rates are used, often together with detector characterization data and acceptable knowledge, to solve the expressions for unknown item properties (such as ${ }^{240} \mathrm{Pu}_{\text {eff }}$ mass) [4]. The salient point for the present discussion is that, the on the average, the nature of the time correlated pulse train can be represented by these multiplet rates, and the question then becomes how to extract them from the actual recorded data. For completeness we can write the first three observed multiplet rates as:

$R_{1}=\varepsilon \cdot F \cdot \frac{v_{e 1}}{1 !}$

$w_{2} \cdot R_{2}=w_{2} \cdot \varepsilon^{2} \cdot F \cdot \frac{v_{e 2}}{2 !}$

$w_{3} \cdot R_{3}=w_{3} \cdot \varepsilon^{3} \cdot F \cdot \frac{v_{e 3}}{3 !}$

where $F$ is the primary source decay rate; $v_{e i}$ is the $\mathrm{i}^{\text {th }}$ factorial moment of the neutron multiplicity distribution emerging from the measurement item; $\varepsilon$ is the neutron detection efficiency; and the $w$-factors are the gate utilization factors which depend on the method of extraction used and give the fraction of correlated events in the pulse train which are recorded in the finite gate structure.

One form of coincidence gate based passive neutron multiplicity counting is a method which involves acquiring the so called accidentals histogram by fast accidental sampling (FAS) [2]. This represents an extension of the non-overlapping or contiguous Feynman pulse train sampling approach which leads to the well-known variance-to-mean (VTM) approach to simple neutron coincidence counting (based on the first two multiplet rates only) [5]. Under the FAS data acquisition scheme a gate is opened at a periodic rate much faster than the incoming event rate and the histogram of counts recorded, $B_{i}$, is accumulated. The normalized histogram is formed as follows:

$b_{i}=\frac{B_{i}}{\sum_{i=0}^{i_{\max } B_{i}}}$, such that $\sum_{i=0}^{i_{\max }} b_{i}=1$ 
This histogram is then evaluated to quantify its non-random character by various statistical metrics, these in turn are usually related to the expectation values predicted by the point model expressions and used in the numerical solution to obtain assay estimates. For simplicity we shall not discuss here various consistency or outlier tests which might be applied in practice. As described the FAS method oversamples the pulse train because it uses overlapping gates. In this way all of the information in the pulse train is being used, which improves precision, but as a consequence the elements of the histogram are correlated. Derived quantities remain fair estimates of the statistical properties of the pulse train, however, because the overlapping gates remain random with respect to the incoming pulses - we are just counting them more than once but still in the correct proportions. To properly estimate the counting precision and the associated covariance structure in the data it is common practice to divide an assay measurement into a series of shorter acquisition periods and subject the repetitions to direct statistical analysis. In [2] the $b$-histogram is referred to as the random trigger interval (RTI) or accidentals histogram. These terms will be familiar to the measurement community that works with the shift register logic approach which is currently the most commonly fielded approach used for nuclear safeguards missions [6]. But they are just labels, the important observation is that the histogram is formed from a random (with respect to the arrival time of events) sampling of the pulse train using a counting gate of fixed width. In this respect it is the same kind of histogram that SVM approach [1] has to work with. In particular it means that the gate utilization factors will be the same no matter how we decide to extract the statistical metrics that describe the histogram.

Following [2] we define reduced factorial moments as follows:

$$
\begin{aligned}
& m_{1}=\frac{1}{1 !} \cdot \sum_{i=0}^{i_{\max }} i \cdot b_{i}=\frac{1}{1 !} \cdot\langle i\rangle \\
& m_{2}=\frac{1}{2 !} \cdot \sum_{i=0}^{i_{\max }} i \cdot(i-1) \cdot b_{i}=\frac{1}{2 !} \cdot\langle i(i-1)\rangle=\frac{\left\langle i^{2}\right\rangle-\langle i\rangle}{2} \\
& m_{3}=\frac{1}{3 !} \cdot \sum_{i=0}^{i_{\max }} i \cdot(i-1) \cdot(i-2) \cdot b_{i}=\frac{1}{3 !} \cdot\langle i(1-1)(i-2)\rangle=\frac{\left\langle i^{3}\right\rangle-3 \cdot\left\langle i^{2}\right\rangle+2 \cdot\langle i\rangle}{6}
\end{aligned}
$$

where we have introduced the braket notation, \langle\rangle , to denote the histogram (sample) average.

According to [2] the multiplet rates may be expressed in terms of the reduced factorial moments of the $b$-histogram as follows: 
$R_{1}=\frac{1}{T_{g}} \cdot m_{1}$

$w_{2} \cdot R_{2}=\frac{1}{T_{g}} \cdot\left(m_{2}-\frac{m_{1}^{2}}{2}\right)$

$w_{3} \cdot R_{3}=\frac{1}{T_{g}} \cdot\left(m_{3}-m_{2} \cdot m_{1}+\frac{m_{1}^{3}}{3}\right)$

where $T_{g}$ is the duration (width) of the time gate.

By direct substitution we can re-express the multiplet rates in terms of the mean, mean square, and mean cube estimates of the histogram as follows:

$R_{1}=\frac{1}{T_{g}} \cdot\langle i\rangle$

$w_{2} \cdot R_{2}=\frac{1}{2 \cdot T_{g}} \cdot\left[\left\langle i^{2}\right\rangle-\langle i\rangle^{2}-\langle i\rangle\right]$

$w_{3} \cdot R_{3}=\frac{1}{6 . T_{g}} \cdot\left[\left\langle i^{3}\right\rangle+2 \cdot\langle i\rangle^{3}-3 \cdot\langle i\rangle \cdot\left\langle i^{2}\right\rangle+2 \cdot\langle i\rangle\right]$

In [1] central moments formed about the mean are used as statistical metrics instead. The first three central moments are the mean, variance, and skewness which we shall denote by $\mu, \sigma^{2}$ and $\gamma$ respectively and which are evaluated as follows:

$\mu=\sum_{i=0}^{i_{\max }} i \cdot b_{i}$

$\sigma^{2}=\sum_{i=0}^{i_{\max }}(i-\langle i\rangle)^{2} \cdot b_{i}$

$\gamma=\sum_{i=0}^{i_{\max }}(i-\langle i\rangle)^{3} \cdot b_{i}$

By inspection the expressions for the mean, variance and skewness lead to expressions in terms of the mean, mean square and mean cube values and so it obvious that they may in turn themselves be used to express the reduced factorial moments. Perhaps this is most clearly 
illustrated however by using a translation of origin and a back substitution in the expressions for the factorial moments.

$$
\begin{aligned}
& m_{1}=\frac{1}{1 !} \cdot \sum_{i=0}^{i_{\max }} i \cdot b_{i}=\mu \\
& m_{2}=\frac{1}{2 !} \cdot \sum_{i=0}^{i_{\max }}([i-\mu]+\mu) \cdot([i-\mu]+(\mu-1)) \cdot b_{i}=\frac{\sigma^{2}+\mu(\mu-1)}{2} \\
& m_{3}=\frac{1}{3 !} \cdot \sum_{i=0}^{i_{\max }}([i-\mu]+\mu) \cdot([i-\mu]+(\mu-1)) \cdot([i-\mu]+(\mu-2)) \cdot b_{i}=\frac{\gamma+3(\mu-1) \sigma^{2}+\mu(\mu-1)(\mu-2)}{6}
\end{aligned}
$$

By back substitution we therefore arrive at the following alternative but equivalent expressions for the multiplet rates:

$$
\begin{aligned}
& R_{1}=\frac{1}{T_{g}} \cdot \mu \\
& w_{2} \cdot R_{2}=\frac{1}{2 \cdot T_{g}} \cdot\left(\sigma^{2}-\mu\right) \\
& w_{3} \cdot R_{3}=\frac{1}{6 . T_{g}} \cdot\left(\gamma-3 \cdot \sigma^{2}+2 \mu\right)
\end{aligned}
$$

The results for the second multiplet rate is recognized in relation to the Feynman VTM metric mentioned above.

To conclude we have shown that the SVM and RTI approaches described in [1] and [2], respectively, are mathematically equivalent and from an application standpoint are the same because they are based on the same theoretical foundations when interpreted through the pointmodel expressions. In principle any number of third order polynomials could be used to extract the correlated information from the pulse train, but the factorial moments, SVM, mean-squarecube are as simple, direct, and reliable as any possible alternatives. There seems to be no additional benefit in exploring other alternative approaches as it would not result in a new technique. It is worth noting that the mean value is expected to equal the average event rate multiplied by the duration of the gate. In current practice this product might reasonably range from $10 \mathrm{~Hz}$ times $8 \mu \mathrm{s}$ (mean $=80 \times 10^{-6}$ counts) to $10 \mathrm{MHz}$ and $128 \mu$ s (mean of 1280 counts in the gate). This numerical range is not wide by modern standards of computing machine 
precision and so whichever of these three analysis methods one picks the estimates of the multiplet rates should remain robust. The use of central moments over reduced factorial moment appears less appealing when extensions to higher multiplets such as the fourth and fifth order are considered, just because the algebraic expressions don't simplify as nicely. Note that the two other techniques (signal triggered and mixed histogram analysis) described in [2] and evaluated experimentally in [7] remain different in character because they make use of event triggered histogram data either in isolation or in combination with the random triggered histogram.

\section{ACKNOWLEGEMENTS}

This work was sponsored by the U.S. Department of Energy (DOE), National Nuclear Security Administration (NNSA), Office of Nonproliferation Research and Development (NA-22).

\section{References}

[1]. C Dubi, T Ridnick, I Israelashvili, J Bagi, and J Huszti, A method for the estimation of fissile mass by measuring the number of neutron signals within a specific time interval, Nucl Instrum and Meths in Phys Res A(673)(2012)111-115.

[2]. S Croft, D Henzlova, and DK Hauck, Extraction of correlated count rates using various gate generation techniques: Part I Theory, Nucl Instrum and Meths in Phys Res A(691)(2012)152-158.

[3]. K Böhnel, The effect of multiplication on the quantitative determination of spontaneously fissioning isotopes by neutron correlation analysis, Nucl Sci and Engin 90(1985)75-82.

[4]. I Pázsit, A Enqvist, and L Pál, A note on the multiplicity expressions in nuclear safeguards, Nucl Instrum and Meths in Phys Res A(603)(2009)541-544.

[5]. S Croft, A Favalli, DK Hauck, D Henzlova, and PA Santi, Feynman variance-to-mean in the context of passive neutron coincidence counting, Nucl Instrum and Meths in Phys Res A(686)(2012)136-144.

[6]. N Ensslin, WC Harker, MS Krick, DG Langner, MM Pickrell, and JE Stewart, Application guide to neutron multiplicity counting, Los Alamos National Laboratory LA-13422-M(1998).

[7]. D Henzlova, S Croft, HO Menlove, and MT Swinhoe, Extraction of correlated count rates using various gate generation techniques: Part II Experiment, Nucl. Instrum. and Meths in Phys Res A(691)(2012)159-167. 